Apall manines

April 6-12. UCLA Symposium on Interferons as Cell Growth Inhibitors and Antitumor Factors. Steamboat Springs, CO. Info: Betty Handy, UCLA Symposia, Molecular Biology Institute, University of California, Los Angeles, CA 90024

April 6-13. UCLA Symposium on Growth Factors, Tumor Promoters, and Cancer Genes. Steamboat Springs, CO. Info: See for April 612.

April 6-13. UCLA Symposium on Transcriptional Control Mechanisms. Keystone, CO. Info: See for April 612.

April 7-8. Commercial Opportunities in Biopolymers. London. Info: Online International, Pinner Green House, Ash Hill Dr., Pinner, Middlesex HA5 2AE, U.K.

April 7-10. Energy from Biomass and Wastes. Washington, D.C. Info: Marianne MacDonald, Institute of Gas Technology, 3424 South State Street, Chicago, IL 60616

April 8-10. New Frontiers in the Study of Gene Functions. Valley Forge, PA. Info: Buff Biddle, Symposium Coordinator, Smith Kline \& French Laboratories, P.O. Box 7929, Philadelphia, PA 19101

April 8-10. International Symposium on Monoclonals and DNA Probes in Diagnostic and Preventive Medicine. Florence, Italy. Info: Fondazione Giovanni Lorenzini, Via Monte Napoleone, 23, 20121 Milan, Italy

April 14-17. Society for General Microbiology Symposium: "Regulation of Gene Expression-25 Years On." Warwick, U.K. Info: Meetings Assistant, Society for General Microbiology, Harvest House, 62 London Road, Reading, Berks RGI 5AS, U.K.

April 15-17. International Conference of Bioreactor Fluid Dynamics. Cambridge, U.K. Info: Conference Organizer, Bioreactor Fluid Dynamics, BHRA, The Fluid Engineering Centre, Cranfield, Bedford MK43 0A], U.K.
April 28-29. ATCG Biotechnology Patent Conference. Rockville, MD. Info: David Grounds, Education Coordinator, American Type Culture Collection, 12301 Parklawn Dr., Rockville, MD 20852

April 29-May 1. Bio Expo 86. Boston, MA. Info: Conference Director, Bio Expo 86, Cahners Exposition Group, 999 Summer St., P.O. Box 3833, Stamford, CT 06905-9990

\section{MYY meztines}

May 13-15. Biotech 86 Europe. London. Info: Online International, Pinner Green House, Ash Hill Drive, Pinner, Middlesex, HA5 2AE, U.K.

May 13-16. 8th Symposium on Biotechnology for Fuels and Chemicals. Gatlinburg, TN. Info: Charles D. Scott, Oak Ridge National Laboratory, P.O. Box X, Oak Ridge, TN 37831

\section{InT Mathines}

June 16-19. 3rd International Conference on Biotechnology in the Pulp and Paper Industry. Stockholm, Sweden. Info: K-E. Eriksson, Svenska Traeforskningsinstitutet Box 5604, S11486 Stockholm, Sweden

June 23-27. International Symposium on Immobilized Enzymes and Cells. Waterloo, Ont. Info: The Director, Industrial Biotechnology Centre, University of Waterloo, Waterloo, Ont., Canada N2L 3GI

\section{DEAIIT CHNiex}

Calgene (Davis, CA) elected its president, Roger Salquist, to the additional post of chief executive officer. He succeeds founder Norman Goldfarb, who remains chairman of the board.

Mesa Diagnostics (Los Alamos, NM) named R. Darrell Phelps president and chief executive officer.

\section{ADVERTISIHG SALES/SALES OFFICES NORTY AMERICAM SALES MAHAGER}

Marion Delaney

\section{MEW YORK}

Marion Delaney,

Sales Manager, (212) $477-9600$

65 Bleecker Street

New York. NY 10012

\section{EAST COAST}

Denis O'Malley, (212) $477-9623$

65 Bleecker Street

New York, NY 10012

SOUTHEAST

Marion Delaney, (212) 477.9600

65 Bleecker Street

New York, NY 10012

\section{MIDWEST}

Didier \& Broderick, (312) 498-4520

255 Revere Drive Suite 105/106

Northbrook, IL 60062

\section{SOUTHWEST}

Bill Powell, (713) 376-2368

18103 Mahogany forest Dr.

Spring, TX 77379

\section{WEST COAST}

Gretchen Hasse, (415) 392-6794

57 Post Street, Suite 712.715

San Francisco, CA 94104

Dave Heidersbach, (818) 796-9200

119 West Bellevue Drive

Pasadena, CA 91105

canaba

Peter Drake, (416) 690-2423

17 Pine Crescent

loronto, Ontario

M4E IL1 Canada

UNITED KILGDOW

Andy Sutherland, (01) 836-6633

4 Little Essex Street

London WC2R 3LF
EUROPEAH ADVERTISING MANAGER

Andy Sutherland
W. GERMAWY \& AUSTRIA

Franz Schrecklinger, TMW Top Media

Werbegesellschaft mbh,

Eppsteiner Strasse 36

$D 6(000$ Frankfurt am Main 1

Telephone (69) 726046

Telex 413759

\section{SCANDINAVIA}

Andrew Karnig, Andrew Karnig \& Associates AB

Fimnbodavagen 1

13131 NACKA

Telephome (0)8) 440005

Telex 89101755

\section{HOLLAMD \& BELGIUM}

Adele Struyck, Astra

Postbus 18

$3712 \mathrm{ZG}$ Huis ter Heide

The Netherlands

Telephone 0340423265

JAPAN

Mashy Yoshikawa, Orient Echo lac.

1101 Grand Maison

Shimomiyabi-cho 8

Shinjuku-ku, 'lokyo 162

Japan

Telephone 03-235-5961

Telex I33376 MYORIENT

\section{FRANCE}

Françoise Teisseyre,

8 Rue Barye

75017 Paris

Telephone (1) 46225154

australla

Gus Bartel, (ius Bartel \& Associates

P() 1755 Southport

Queensland 4215

Telephone 075466577

Telex 40472-BR 433 